\title{
Gauss 自由场及其相关领域
}

献给钱敏教授 90 华诞

\author{
陈大岳，章复喜* \\ 北京大学数学科学学院, 北京 100871 \\ E-mail: dayue@math.pku.edu.cn, zhangfxi@math.pku.edu.cn \\ 收稿日期: 2017-06-14；接受日期: 2017-10-30；网络出版日期: 2017-11-28; * 通信作者 \\ 国家自然科学基金 (批准号: 11531001, 11528101 和 11371040) 资助项目
}

摘要 本文综述 Gauss 自由场近几年的发展, 包括各种等价定义、优美的性质和精细的结构, 并介绍 相关的 Liouville 量子引力场模型 (随机曲面), 包括 Liouville 测度和该曲面上的标准 Brown 运动.

关键词 Gauss 自由场 对数相关的 Gauss 场 Liouville 量子引力场

MSC (2010) 主题分类 $60 \mathrm{G} 60,60 \mathrm{~K} 35$

\section{1 引言}

$d$ 维 Gauss 自由场是以 $d$ 维 Brown 运动的 Green 函数为协方差的零均值 Gauss 场, 它被称为 $d$ 维时间参数、一维空间取值的 Brown 运动 (即噪声), 是 $\mathbb{R}^{d}$ 中的调和函数最自然的扰动. 正如 Brown 运动是众多随机过程 (视为时间参数的随机函数) 在时空尺度变化下的极限, Gauss 自由场是随机多米 诺贴砖 (domino tiling) ${ }^{[1]}$ 和随机矩阵的特征多项式 ${ }^{[2]}$ 等众多概率模型中的随机函数的极限. 也正如 Brown 运动, Gauss 自由场的内在结构相当丰富. 它有诸如马氏性和共形不变性等优美的数学性质, 并 表现出诸如分形等复杂的数学现象. Gauss 自由场有着广泛的应用前景, 特别是, 基于 Gauss 自由场的 Gauss 乘法噪声在一维情形可刻画金融中的价格波动, 在二维情形则是统计物理中量子场论的出发点.

相应地, 离散型 Gauss 自由场的协方差是随机游动的 Green 函数. Gauss 自由场和离散型 Gauss 自由场在最近 10 年的发展如火如茶, 相关文献汗牛充栋. 本文引用的仅是作者认为比较具有代表性的 一些文献, 远不足以表达该领域发展之迅速.

虽然本文重点关注二维 Gauss 自由场, 但是我们在定义时仍尽量进行一般性介绍. 第 2 节介绍离 散型 Gauss 自由场的定义, 场的最大值、水平集和极值点集等精细结构, 对应的首达渗流模型中距离 指数与测地线维数, 以及它与其他概率分支的联系. 第 3 节介绍 Gauss 自由场的定义, 马氏性、厚点 和共形不变性等性质, 相关的 Liouville 量子引力场, 以及一维 Gauss 自由场和高维 Gauss 自由场的相 关研究. 


\section{2 离散型 Gauss 自由场}

为简单起见, 我们省略 “离散型” 字样. 在本节中, Gauss 自由场均指离散型 Gauss 自由场.

\section{1 电网络上的 Gauss 自由场}

Gauss 自由场源自统计物理, 可以刻画测量海拔的统计模型: 假设 $V$ 是有限集; 已知 $v_{0} \in V$ 处的 海拔; 目标是测量 $V$ 中所有位置的海拔; 采用的方法是测量 $V$ 中某些点对 $e=\{u, v\} \in E$ 中两点之间 的海拔差, 本文中的点对都指 $u$ 与 $v$ 无序, 且 $v \neq u$. 假设对任意 $e \in E$, 测量出来的海拔差与真实海 拔差之间的差异 (记为 $Z(e)$ ) 是一个独立的随机变量, 服从 $N\left(0, \sigma(e)^{2}\right)$, 其中方差 $\sigma(e)^{2}$ 为已知, (零均 值表示测量没有受到因海拔而产生的重力影响; 测量误差的方差仅依赖于测量办法), 那么, 海拔的测 量值与真实值之间的误差的最大似然估计 $\left\{\xi_{v}\right\}_{v \in V}$ 称为 Gauss 自由场 (Gaussian free field) 或无重调 和晶体 (massless harmonic crystal). 具体地, 对任意 $V$ 上满足 $\alpha_{v_{0}}=0$ (因为 $v_{0}$ 处的海拔为已知, 因 此, 测量误差为 0$)$ 的向量 $\alpha=\left\{\alpha_{v}\right\}_{v \in V}$, 似然函数为

$$
L(\alpha)=\frac{1}{\prod_{e \in E} \sqrt{2 \pi \sigma(e)^{2}}} \exp \left(-\frac{1}{2 \sigma(e)^{2}} \sum_{e \in E}(Z(e)-\nabla \alpha(e))^{2}\right),
$$

其中, 对任一条边 $e$ 指定一个方向, 将其两个顶点分别记为 $e_{-}$和 $e_{+}$, 然后定义 $\nabla \alpha(e)=\alpha_{e_{+}}-\alpha_{e_{-}}$. 注意到 $\mathbb{E} Z(e)=0$, 所以, Gauss 自由场的分布不依赖于边的方向. 令

$$
Q(\alpha)=\sum_{e \in E} \frac{1}{\sigma(e)^{2}}(Z(e)-\nabla \alpha(e))^{2},
$$

那么, $Q(\alpha)$ 的最小值点 (即似然函数的最大值点) 就是 Gauss 自由场, 记为 $\xi$. 根据变分公式, Gauss 自由场 $\xi$ 满足: 若 $\alpha_{v_{0}}=0$, 则

$$
\sum_{e \in E} \frac{1}{\sigma(e)^{2}}(Z(e)-\nabla \xi(e)) \nabla \alpha(e)=0 .
$$

下面从电网络的角度给出 Gauss 自由场的定义. 首先给出电网络的定义. 假设 $V$ 是一个可数集, $E$ 是 $V$ 中某些无序点对组成的集合, 使得 $(V, E)$ 成为一个连通的简单图. 假设 $c: E \rightarrow(0, \infty)$. 若将 $e$ 视为导线, 则 $c(e)$ 表示 $e$ 的电导, 即电阻 $r(e)$ 的倒数. 若 $e=\{u, v\} \in E$, 则称 $u$ 与 $v$ 之间有边相连, 此时, 称它们为邻居, 记 $u \sim v$, 也记 $c_{u, v}=c(e)$. 称 $(V, E, c)$ 为一个电网络. 电网络 $(V, E, c)$ 对应着一 个互通的可配称马氏链, 其中, 可配称指存在配称测度. 将该马氏链记为 $\left\{S_{n}: n \geqslant 0\right\}$, 其转移概率定 义为 $p_{u, v}=c_{u, v} / c_{u}$, 其中 $c_{u}=\sum_{w \sim u} c_{u, w} .\left\{c_{u}\right\}_{u \in V}$ 就是配称测度. 反过来, 任意互通的可配称马氏链 都对应着一个电网络: 假设转移概率 $\left(p_{u, v}\right)_{u, v \in V}$ 满足 $p_{u, u}=0$, 那么令 $E=\left\{\{u, v\}: p_{u, v}>0\right\}$; 取一 个配称测度 $\left\{c_{u}\right\}_{u \in V}$, 并令 $c_{u, v}=c_{u} p_{u, v}$ 即可. 因此, 电网络与互通的可配称马氏链是等价的. 特别地, 当 $c(e) \equiv 1$ 时, 对应的马氏链就是图 $(V, E)$ 上的简单随机游动, 我们也将上述可配称马氏链称为随机 游动.

假设 $V$ 有限. 取 $v_{0} \in V$, 称之为边界, 记 $\partial=\left\{v_{0}\right\}, V^{o}=V \backslash \partial$. 令

$$
G(u, v)=\frac{1}{c_{v}} \mathbb{E}_{u} \sum_{n=0}^{\tau-1} 1_{\left\{S_{n}=v\right\}}, \quad \text { 其中 } \tau=\inf \left\{n \geqslant 0: S_{n} \in \partial\right\} .
$$

并称之为该电网络的 (边界截杀的) Green 函数. 可以证明, $G(\cdot, \cdot)$ 是对称正定函数, 因此在视为矩阵 后可以作为 Gauss 随机向量的协方差矩阵. 
定义 1 若 $\xi=\left\{\xi_{v}\right\}_{v \in V}$ 为零均值的 Gauss 随机向量, 满足 $\mathbb{E} \xi_{u} \xi_{v}=G(u, v), \forall u, v \in V$, 则称 $\xi$ 为 $V$ 上的 Gauss 自由场.

需要注意的是, 当 $u=v_{0}$ 或 $v=v_{0}$ 时, $G(u, v)=0$, 因此, Gauss 自由场满足 $\xi_{v_{0}}=0$, 即它满足 Dirichlet 边界条件.

下面从不同的角度刻画 Gauss 自由场的分布, 结论罗列在定理 2 中, 在补充定义 $\xi_{v_{0}}=0$ 后, 它 们都可作为 Gauss 自由场的等价定义. 根据定义 1 , 为指出 $\left.\xi\right|_{V \backslash\left\{v_{0}\right\}}$ 的联合密度函数, 我们只需求出 $G(\cdot, \cdot)$ 的逆矩阵即可, 于是得到定理 $2(\mathrm{i})$. 如果对每条边指定一个方向, 当我们考虑从 $u$ 到 $v$ 的单位 电流 $I_{u, v}$ 时, 它在边 $e$ 上的取值 $I_{u, v}(e)$ 可能为非负 (若电流在 $e$ 上从 $e_{-}$流向 $e_{+}$), 也可能为负 (若 不然). 令 $I_{u, u}(e) \equiv 0$. 在海拔测量模型中, 令 $r(e)=\sigma(e)^{2}$. 那么, 用电流的语言解释 $(2.1)$, 就是定 理 2(iii). 最后, 令 $R_{u, v}=\sum_{e \in E} I_{u, v}(e)^{2} r(e)$, 它表示 $u$ 与 $v$ 两点之间的有效电阻, 即单位电流所做的 功. 那么, 我们还可以用有效电阻来定义 Gauss 自由场, 即定理 2(ii).

定理 2 (参见文献 $[3,(1.2 .3)]$ 和 [4, 命题 2.24 和 (2.25)]) 假设 $\xi=\left\{\xi_{v}\right\}_{v \in V}$ 是 Gauss 自由场; $Z(e)(e \in E)$ 相互独立, $Z(e) \sim N(0, r(e))$, 则下面三条成立:

(i) $\left.\xi\right|_{V^{o}}$ 的联合密度函数为 $\rho(h) \propto \exp \left(-\frac{1}{2} \sum_{u \sim v} c_{u, v}\left(h_{u}-h_{v}\right)^{2}\right)$, 其中, 补充定义 $\left.h\right|_{\partial}=0$;

(ii) $\mathbb{E}\left(\xi_{u}-\xi_{v}\right)^{2}=R_{u, v}$;

(iii) 令 $\eta_{v}=\sum_{e \in E} I_{v_{0}, v}(e) Z(e)$, 则 $\eta=\left\{\eta_{v}\right\}_{v \in V}$ 是 Gauss 自由场.

一般地, 我们还可以将 $V$ 的非空真子集 $\partial V$ 视为 $v_{0}$, 记 $V^{o}=V \backslash \partial V$, 考虑 $V^{o} \cup\{\partial V\}$, 并对任意 $u \in V^{o}$ 补充定义 $c(u, \partial V)=\sum_{v \in \partial V} c(u, v)$ 即可. 假设 $u \in V^{o}, v \neq u$, 则有 $G(u, v)=\sum_{w \sim u} p_{u, w} G(w, v)$, 因此, $Z:=\xi_{u}-\sum_{w \sim u} p_{u, w} \xi_{w}$ 与 $\left.\xi\right|_{V \backslash\{u\}}$ 相互独立. 注意到 $\mathbb{E} Z=0$, 且

$$
\mathbb{E} Z^{2}=\mathbb{E} Z\left(\xi_{u}-\sum_{w \sim u} p_{u, w} \xi_{w}\right)=\mathbb{E} Z \xi_{u}=G(u, u)-\sum_{w \sim u} p_{u, w} G(w, u)=\frac{1}{c_{u}} .
$$

对所有 $U \subset V$, 令 $U^{o}=\left\{v \in U \cap V^{o}: w \in U, \forall w \sim v\right\}, \partial U=U \backslash U^{o}$. 特别地, 取 $U=\{u\} \cup\{w: w \sim u\}$, 于是 $U^{o}=\{u\}$, 则由 $\xi_{u}^{U}=Z, \xi_{w}^{U}=0, \forall w \in \partial U$ 定义的 $\xi^{U}$ 是 $U$ 上的 Gauss 自由场. 补充定义 $\left.\xi^{U}\right|_{V \backslash U}=0$. 那么,

$$
\xi=\xi^{U}+\eta \text {, 其中 } \eta \text { 为 }\left.\xi\right|_{V \backslash U^{o}} \text { 的调和延拓, 并且 } \xi^{U} \text { 与 } \eta \text { 相互独立. }
$$

上式中的 “调和延拓” 指 $\left.\eta\right|_{V \backslash U^{o}}=\left.\xi\right|_{V \backslash U^{o}}$, 且 $\eta_{u}=\sum_{v \in \partial U} \mathbb{P}_{u}\left(S_{\tau}=v\right) \xi_{v}, \forall u \in U^{o}$, 其中 $\tau$ 为随机 游动 $\left\{S_{n}: n \geqslant 0\right\}$ 首达 $\partial U$ 的时间. 事实上, (2.2) 对所有 $U \subset V$ 成立. 如果将顶点视为时间, $U^{c} 、 \partial U$ 和 $U^{o}$ 分别视为过去、现在和将来, 那么 (2.2) 就是马氏性. 马氏性可以说是研究 Gauss 自由场的出发 点, 因此该领域的文献都会讲述, 如文献 [5, 定理 1.17]. 根据马氏性知, Gauss 自由场的调和平均值关 于时间具有独立增量性, 其增量服从零均值的正态分布.

定理 3 (马氏性) 假设 $\xi=\left\{\xi_{v}\right\}_{v \in V}$ 是 Gauss 自由场, 则 (2.2) 对任意 $U \subset V$ 成立.

事实上, 上面的结论对常返的电网络 (即其对应的随机游动常返) 都适用, 只需要合理设置边界使 得边界截杀的 Green 函数有限. 下面考虑非常返情形. 假设 $V$ 可列, 且电网络非常返. 令

$$
G(u, v)=\frac{1}{c_{v}} \mathbb{E}_{u} \sum_{n=0}^{\infty} 1_{\left\{S_{n}=v\right\}},
$$

则 $V$ 上的 Gauss 自由场 $\xi=\left\{\xi_{v}\right\}_{v \in V}$ 是一个零均值的 Gauss 系, 满足 $\mathbb{E} \xi_{u} \xi_{v}=G(u, v)$. 一个特殊例子 是分支随机游动 (branching random walk). 下面介绍这个模型. 将带根点 $o$ 的树记为 $T$, 若 $u \sim v$ 且 $u$ 
在连接 $v$ 与 $o$ 的 (唯一的) 路径上, 则称 $u$ 为 $v$ 的父结点, $v$ 为 $u$ 的子结点. 令 $c(e) \equiv 1$. 考虑其上的 Gauss 自由场 $\xi=\left\{\xi_{v}\right\}_{v \in T}$. 由定理 $2(\mathrm{i})$ 知, $\{\nabla \xi(e)\}_{e \in E}$ 是独立同分布的随机变量, 且 $\nabla \xi(e) \sim N(0,1)$. 在齐次树 $T=T^{d}$ 中, 每个顶点都有 $d$ 个子结点, 此时 Gauss 自由场等同于如下模型: 将每个结点视 为一个粒子, 根点表示原始粒子, 它放置在原点, 当每个 (父) 粒子 $u$ 分裂成 $d$ 个 (子) 粒子 $v$ 时, 新生 粒子 $v$ 在父粒子 $u$ 位置的基础上再产生位移 $X(e)=\nabla \xi(e)$, 其中 $e=\{u, v\}$. 于是, 粒子 $v$ 最终位于 $\sum_{e} X(e)$, 求和号中的 $e$ 取连接 $v$ 与 $o$ 的路径上所有的边. 这个模型就是分支随机游动, 在特殊设置 下, 我们可以将分支随机游动对应为平面格点上的一个 Gauss 场, 见注 5. 在更一般的模型中, 新生粒 子的数目可以是随机的, 所有新粒子产生的位移也可以不相互独立, 它们由一个点过程决定.

\section{2 场的精细结构}

本节考虑平面格点 $\left(\mathbb{Z}^{2}, E^{2}\right)$. $\mathbb{Z}^{2}$ 中的两点 $u$ 和 $v$ 之间有边相连, 当且仅当 $u$ 和 $v$ 的欧氏距离 $\|u-v\|=1$. 令 $c(e) \equiv 1 / 4$. 假设 $V \subset \mathbb{Z}^{2}, V$ 的边界定义为 $\partial V=\{u \in V: \exists v \notin V$ 使得 $u \sim v\}$, 内部 为 $V^{o}=V \backslash \partial V$. 将 $V$ 上的 Gauss 自由场记为 $\xi$, 它满足 Dirichlet 边界条件 $\left.\xi\right|_{\partial V}=0$. 基于马氏性, 若 $\eta$ 是 $\partial V$ 上的函数, 我们也将 $\xi$ 叠加 $\eta$ 的调和延拓 (至 $V^{o}$ ) 称为边界为 $\eta$ 的 Gauss 自由场. 如果不 特别说明, 下文中的 Gauss 自由场都指满足 Dirichlet 边界条件. 需要说明的是, 在不同的文献中, 为了 简化常数, Gauss 自由场 (等价地, 电导函数) 可以差一个常数倍数, 即 $\xi$ 如上定义, 那么对所有 $a \neq 0$, $\left\{a \xi_{v}\right\}_{v \in V}$ 都称为 Gauss 自由场.

本节取 $V=V_{N}=\{0,1, \ldots, N-1\}^{2}$, 并介绍 $N \rightarrow \infty$ 时 $V$ 上的 Gauss 自由场 $\xi$ 的极限行为. 首先需要指出的是, $\xi$ 具有分层结构. 我们在此处介绍一种分层方案: 假设 $v$ 不靠近边界 (例如, $v$ 与 $\partial V$ 的距离至少为 $\alpha N, \alpha$ 为常数). 对一列正整数 $\ell_{1}<\ell_{2}<\cdots$, 将以 $v$ 为中心的边长为 $\ell_{r}$ 的 方块记为 $B_{r}(v)$. 当 $B_{r}(v) \subset V$ 时, 令 $\eta_{r, v}$ 为 $\left.\xi\right|_{\partial B_{r}(v)}$ 的调和延拓在 $v$ 的值. 那么, 根据马氏性, $\xi_{v}-\eta_{1, v}, \eta_{1, v}-\eta_{2, v}, \ldots$ 相互独立, 因为它们表示 Gauss 自由场的调和平均值在不同时间区域上的增 量, 将它们依次记为 $\xi_{1, v}, \xi_{2, v}, \ldots$ 特别地, 如果取 $\ell_{r}=(2 K)^{r}$, 其中 $K$ 为给定的正整数, 那么, 通过对 Green 函数的精细估计可知 $\mathbb{E} \xi_{r, v}^{2} \approx C_{K}$, 其中 $C_{K}$ 是依赖于 $K$ 的常数. 于是, $\xi_{v}$ 可分解为相互独立的 零均值正态随机变量之和, 且它们的方差都约为 $C_{K}$. 具体地,

$$
\xi_{v}=\xi_{1, v}+\xi_{2, v}+\cdots+\xi_{m, v}+\eta,
$$

其中 $m=\left\lfloor\log _{2 K}(2 \alpha N)\right\rfloor$, 而 $\eta$ 为可忽略的尾项. 下面的定理 4 和 7 都是基于类似的分解而得到 的. 事实上, 所有对数相关的零均值二维 Gauss 场都有类似的分解 (粗略地讲, 对数相关是指 $\mathbb{E} \xi_{u} \xi_{v}$ $\approx \log N /(|u-v| \vee 1)$ ). 于是下述定理 4(i) 和 4(ii) 对所有对数相关的零均值二维 Gauss 场都成立.

定理 4 (最大值, 参见文献 [6, 定理 2]、[7, 定理 1.2] 和 [8, 定理 1.4]) 假设 $\xi$ 是 $V_{N}$ 上的 Gauss 自由场. 记 $M_{N}=\max _{v \in V_{N}} \xi_{v}$, 则下面的三条成立:

(i) $M_{N} / \log N$ 依概率收敛到某常数 $c_{1}$ (对 Gauss 自由场, $c_{1}=2 \sqrt{2 / \pi}$ );

(ii) $\mathbb{E} M_{N}=m_{N}+O(1)$, 其中 $m_{N}=c_{1} \log N-c_{2} \log \log N$ (对 Gauss 自由场, $c_{2}=(3 / 4) \sqrt{2 / \pi}$ );

(iii) $M_{N}-\mathbb{E} M_{N}$ 依分布收玫, 其极限分布函数形如 $F(x)=\mathbb{E} \exp \left(-Z \mathrm{e}^{-2 \sqrt{2} x}\right)$, 其中 $Z$ 是随机 变量.

注 5 考虑齐次树 $T^{4}$ 上的分支随机游动. 将每个粒子视为一个方块, 当父粒子分裂成 4 个子粒 子时, 我们对方块进行田字格的剖分, 将剖分后的每个小方块对应一个子粒子. 将第 $n$ 代粒子的位置 赋于它对应的小方块的中心, 我们便得到 $V_{N}$ 中的一个零均值的 Gauss 场 (其中 $N^{2}=4^{n}$ ), 仍然称为 
分支随机游动. 显然, 分支随机游动具有 (2.3) 中的分层结构 (对应地, $m=n=\log _{2} N, \eta=0$ ). 对于更 一般的分支随机游动, 关于粒子位置的极值也有类似结论 (参见文献 [9]).

下面介绍 Gauss 场的水平集 (level set) $U_{\alpha}$ 和极值点集 $E_{r}$. 令

$$
U_{\alpha}:=\left\{u \in V_{N}: \xi_{u} \geqslant \alpha \mathbb{E} M_{N}\right\}, \quad E_{r}:=\left\{u \in V_{N}: \xi_{u} \geqslant \xi_{v}, \forall v \text { 满足 }\|v-u\|_{1} \leqslant r\right\},
$$

其中 $\|v-u\|_{1}=\left|v_{1}-u_{1}\right|+\left|v_{2}-u_{2}\right|, \forall\left(v_{1}, v_{2}\right),\left(u_{1}, u_{2}\right) \in \mathbb{Z}^{2}$.

定理 6 (水平集和极值点集, 参见文献 [10, 定理 1.6]、[11, 定理 1.3] 和 [12, 定理 1.1]) 假设 $0<\alpha<1$. 当 $N \rightarrow \infty$ 时, $r \rightarrow \infty$ 且 $r / N \rightarrow 0$, 那么下面两条成立:

(i) $\left(\log \left|U_{\alpha}\right|\right) / \log N$ 依概率收敛于 $2\left(1-\alpha^{2}\right)$;

(ii) 令 $\eta(A \times B):=\mid\left\{u \in E_{r}: u / N \in A\right.$ 且 $\left.\xi_{u}-m_{N} \in B\right\} \mid$, 它描述特定高度对应的极值点集, 则 $\eta$ 依分布收敛于 $[0,1]^{2} \times \mathbb{R}$ 上密度为 $Z(d x) \otimes \mathrm{e}^{-\sqrt{2 \pi} h} d h$ 的 Poisson 点过程, 其中 $Z(d x)$ 是 $[0,1]^{2}$ 上的一 个随机测度.

取定 $\gamma>0$. 对于 $V$ 中的任意路径 $P$, 令

$$
W_{\gamma}(P):=\sum_{z \in P} \mathrm{e}^{\gamma \xi_{z}}, \quad d_{\gamma}(u, v):=\min \left\{W_{\gamma}(P): P \text { 为连接 } u \text { 和 } v \text { 的路径 }\right\},
$$

这个模型称为 Liouville 首达渗流 (first passage percolation). 注意到 $\xi$ 是连续型随机向量, 几乎必然存 在唯一的 (连接 $u$ 和 $v$ 的) 路径 $P$ 使得 $W_{\gamma}(P)=d_{\gamma}(u, v)$, 该路径称为测地线, 记为 $\mathrm{GeO}_{u, v}$. 下面的结 论刻画 Liouville 首达渗流模型的距离指数和测地线维数.

定理 7 (距离指数和测地线维数, 参见文献 [13, 定理 1.3] 和 [14, 定理 1.1]) 当 $\gamma$ 充分小时, 下面 两条成立:

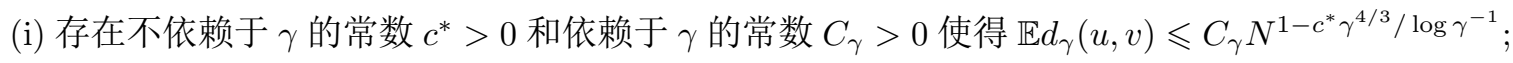

(ii) 存在 $\alpha_{\gamma}>0$ 使得对任意 $\kappa \in(0,1)$, 有 $\mathbb{P}$ (若 $\|u-v\| \geqslant \kappa N$, 则 $\left.\left|\mathrm{Geo}_{u, v}\right|>N^{1+\alpha_{\gamma}}\right) \rightarrow 1$.

\subsection{Gauss 自由场与其他概率分支的联系}

假设 $G=(V, E)$ 是一个电网络, $v_{0} \in V$. 令 $\partial V=\left\{v_{0}\right\},\left\{\eta_{v}\right\}_{v \in V}$ 是 Gauss 自由场, 满足 Dirichlet 边界 $\eta_{v_{0}}=0$. 假设 $X=\left\{X_{t}\right\}_{t \geqslant 0}$ 是 $V$ 上从 $v_{0}$ 出发的 (连续时间参数的) 随机游动, 与 $\xi$ 相互独立.

定理 8 (与随机游动的局部时, 参见文献 [15, 定理 1.1]、[16, 定理 3] 和 [17, 定理 3.1]) 令 $\mathcal{L}_{s}(v)$ 为 $X$ 在 $v$ 的局部时, 令 $\tau(t)=\inf \left\{s \geqslant 0: \mathcal{L}_{s}\left(v_{0}\right) \geqslant t\right\}$, 则下面两条成立:

(i) (第二 Ray-Knight 定理) $\left\{\mathcal{L}_{\tau_{t}}(v)+\frac{1}{2} \eta_{v}^{2}\right\}_{v \in V} \stackrel{d}{=}\left\{\frac{1}{2}\left(\eta_{v}+\sqrt{2 t}\right)^{2}\right\}_{v \in V}$;

(ii) $\left\{\sqrt{\mathcal{L}_{\tau_{t}}(v)}\right\}_{v \in V} \preceq \frac{1}{\sqrt{2}}\left\{\left(\eta_{v}+\sqrt{2 t}\right) \vee 0\right\}_{v \in V}$ ，其中 $\preceq$ 表示存在一个耦合使得前者小于后者.

另外, Gauss 自由场还可刻画随机游动的覆盖时的集中性 ${ }^{[17]}$.

假设 $D$ 是 $\mathbb{R}^{2}$ 中的一个 (有界单连通光滑边界的) 区域, 边界分为 $\partial_{+}$和 $\partial_{-}$的两段弧. 将平面进 行边长为 $\varepsilon$ 的正三角形剖分, 那么 $D$ 对应着一个图, 顶点 $V_{\varepsilon}$ 就是在 $D$ 内的三角形顶点, 边则继承三 角形的边. 相应地, $\partial V_{\varepsilon}$ 分为 $\partial_{+, \varepsilon}$ 和 $\partial_{-, \varepsilon}$. 假设 $\left\{\xi_{\varepsilon, v}\right\}_{v \in V_{\varepsilon}}$ 是 Gauss 自由场, 在 $\partial_{+, \varepsilon}$ 和 $\partial_{-, \varepsilon}$ 分别取值 $\lambda$ 和 $-\lambda$, 其中 $\lambda>0$ 不依赖于 $\varepsilon$. 令 $\gamma_{\varepsilon}$ 为 Gauss 值为正、负的分界线.

定理 9 (与 SLE, 参见文献 [18, 定理 1.1]) 存在常值 $\lambda>0$ 使得当 $\varepsilon \rightarrow 0+$ 时 $\gamma_{\varepsilon}$ 依分布收玫于 $\operatorname{SLE}(4)$. 


\section{Gauss 自由场}

\section{1 定义及其性质}

(1) 从随机函数的角度理解 Gauss 自由场. 假设 $D$ 是 $\mathbb{R}^{2}$ 中的一个 (非空单连通开) 区域. 对比离 散型 Gauss 自由场的定义, 从直观上, $D$ 中的 (Dirichlet 边界) Gauss 自由场 $\xi$ 应为一个零均值的随机 函数 $\left\{\xi_{x}\right\}_{x \in D}$, 满足: $\mathbb{E} \xi_{x} \xi_{y}$ 为 $D$ 中 (边界截杀的) Brown 运动的 Green 函数. 具体地, 令 $p_{t}^{D}(x, y)$ 是 $D$ 中 Brown 运动的密度函数, 即 $E_{x} f\left(B_{t}\right) 1_{\left\{\tau_{\partial D}>t\right\}}=\int_{D} f(y) p_{t}^{D}(x, y) d y$ 对任意非负 Borel 函数 $f$ 成立, 其中 $\left\{B_{t}\right\}$ 是 $\mathbb{R}^{2}$ 上的标准 Brown 运动, $\tau_{\partial D}=\inf \left\{t \geqslant 0: B_{t} \in \partial D\right\}$. 令

$$
G_{D}(x, y)=\int_{0}^{\infty} p_{t}^{D}(x, y) d t, \quad G(x, y)=\pi G_{D}(x, y),
$$

其中系数 $\pi$ 是为了使得下式成立:

$$
G(x, y)=\log \frac{1}{\|x-y\|}+C(x, y),
$$

这里 $\|x-y\|$ 是 $x$ 和 $y$ 之间的欧氏距离; $C(x, y)=E_{x} \log \left\|B_{\tau_{D}}-y\right\|$ 是一个有界连续函数, 它关于 $x$ 调 和. 直观上, 我们希望被视为随机函数的 $\xi=\left\{\xi_{x}\right\}_{x \in D}$ 满足 $\mathbb{E} \xi_{x} \xi_{y}=G(x, y)$. 但是, 注意到 $G(x, x)=\infty$, 这表明 $\xi_{x}$ 不是一个随机变量, 即 Gauss 自由场的样本不是一个函数. 然而, 我们仍然可以先把它视为 一个随机函数, 并利用它关于不同测度 $\rho$ 的积分 $\left(X_{\rho}=\int \xi d \rho\right)$ 来刻画它. 将 $D$ 中有紧支撑的符号测 度的全体记为 $\mathcal{M}$, 则直观上，

$$
\mathbb{E} X_{\rho}=0, \quad \mathbb{E} X_{\rho_{1}} X_{\rho_{2}}=\iint_{x, y \in D} G(x, y) \rho_{1}(d x) \rho_{2}(d y)=: \Gamma\left(\rho_{1}, \rho_{2}\right) .
$$

于是, 我们可以认为 Gauss 自由场指的是一个 Gauss 系 $\left\{X_{\rho}: \rho \in \mathcal{M}, \Gamma(\rho, \rho)<\infty\right\}$, 其均值和协方 差由 (3.3) 给出. 需要强调的是, 这样的 Gauss 系存在, 但不存在一个随机函数 $\xi$ 使得 $X_{\rho}:=\int \xi d \rho$ $(\forall \rho \in \mathcal{M})$ 满足 $(3.3)$.

(2) 从随机广义函数的角度理解 Gauss 自由场. 事实上, Gauss 自由场是一个随机广义函数, 它可 视为一阶 Sobolev 空间 $H_{0}^{1}(D)$ 中的标准正态向量, 我们可以通过它对大量函数 $f$ 的积分值 $Y_{f}$ 来表 达它. 具体地, 令

$$
(f, g)_{\nabla}=\int_{D} \nabla f(x) \cdot \nabla g(x) d x, \quad\|f\|_{\nabla}=\sqrt{(f, f)_{\nabla}},
$$

$H_{0}^{1}(D)$ 是 $D$ 中全体光滑紧支撑的函数 (记为 $C_{0}^{\infty}(D)$ ) 在 $\|\cdot\|_{\nabla}$ 下的完备化, 其对偶空间记为 $H_{0}^{-1}(D)$. 取 $H_{0}^{1}(D)$ 的一组标准正交基 $\left\{f_{n}\right\}_{n \geqslant 1}$ 和独立同分布的随机变量序列 $\left\{Z_{n}\right\}_{n \geqslant 1}$, 其中 $Z_{1} \sim N(0,1)$. 令 $\eta_{n}:=\sum_{k=1}^{n} Z_{k} f_{k}$. 对任意 $f=\sum_{n} a_{n} f_{n} \in H_{0}^{1}(D),\left(\eta_{n}, f\right)$ 几乎必然且依 $L^{2}$ 收玫, 其极限记为 $Y_{f}$. 那么,

$$
\mathbb{E} Y_{f}=0, \quad \mathbb{E} Y_{f} Y_{g}=(f, g)_{\nabla}, \quad \forall f, g \in H_{0}^{1}(D) .
$$

特别地, $\left\{Y_{f}, f \in C_{0}^{\infty}(D)\right\}$ 定义了一个随机广义函数 (或符号测度, 简称分布), 将其记为 $\eta=\sum_{n} Z_{n} f_{n}$. 也将 $Y_{f}$ 记为 $\eta(f)=(f, \eta)_{\nabla}$. 需要指出的是, $\eta \notin H_{0}^{1}(D)$, 且 $\eta_{n}$ 在 $H_{0}^{1}(D)$ 中不收玫.

定义 10 称 $\left\{Y_{f}, f \in H_{0}^{1}(D)\right\}$ 或 $\sum_{n} Z_{n} f_{n}$ 为 $D$ 上的 Gauss 自由场.

下面将说明 $\left\{Y_{f}\right\}$ 就是上面的 $\left\{X_{\rho}\right\}$. 假设 $\rho$ 是一个概率分布, $\rho \in H_{0}^{-1}(D)$, 且存在 $f \in H_{0}^{1}(D)$ 使 得 $\rho=-\Delta f$, 于是, $\rho(g)=(f, g)_{\nabla}$ 对 $g \in H_{0}^{1}(U)$ 和 $g=\eta$ 成立. 注意到

$$
f(x)=\frac{1}{2} \int G_{D}(x, y) \rho(d y) .
$$


于是,

$$
(f, f)_{\nabla}=\rho(f)=\int_{D} f(x) \rho(d x)=\frac{1}{2} \iint G_{D}(x, y) \rho(d x) \rho(d y)=\frac{1}{2 \pi} \Gamma(\rho, \rho) .
$$

根据极化方程, $Y_{f}$ 即为上面寻找的 $\frac{1}{\sqrt{2 \pi}} X_{\rho}$, 换言之,

$$
\xi=\sqrt{2 \pi} \eta .
$$

(3) Gauss 自由场的马氏性. 虽然 Gauss 自由场 $\xi$ 不是随机函数 (它在 $x$ 点的 Gauss 值没有意 义), 但是 $\xi$ 在某分布 $\rho$ 下的平均值 $\rho(\xi)$ 是有意义的, 它就是 $\sqrt{2 \pi} Y_{f}$, 其中 $f$ 由 (3.4) 给出. 与离散 型 Gauss 自由场类似, 我们有如下马氏性, 它成立的本质原因是 $H_{0}^{1}(D)=H_{0}^{1}(U)+H_{0}^{1}(U)^{\perp}$, 其中 $H_{0}^{1}(U)^{\perp}=\left\{f \in H_{0}^{1}(D): f\right.$ 在 $U$ 内调和 $\}$.

定理 11 (马氏性, 参见文献 [19, 第 2.6 节]) 假设 $U$ 是 $D$ 的子区域, 则 $\xi=\xi^{U}+\zeta$, 其中 $\xi^{U}$ 与 $\zeta$ 相互独立; $\xi^{U}$ 是 $U$ 上的 Gauss 自由场; $\zeta$ 在 $D \backslash U^{o}$ 上与 $\xi$ 取值一致, 且在 $U$ 中调和.

(4) 二维 Gauss 自由场的性质. 令 $B(x, r)=\{y:\|y-x\| \leqslant r\}$. 记 $\rho_{x, r}$ 是 $\partial B(x, r)$ 上的均匀分布. 令 $\xi_{r}(x)=\rho_{x, r}(\xi)$. 根据 (3.3), 可得

$$
\mathbb{E} \xi_{r}(x) \xi_{r}(x)=\iint G(z, y) \rho_{x, r}(d z) \rho_{x, r}(d y)=\int G(x, y) \rho_{x, r}(d y)=\log \frac{1}{r}+C(x),
$$

其中 $C(x)=E_{x} \log \left\|B_{\tau_{D}}-x\right\|$. 根据定理 11, 令 $r=\mathrm{e}^{-t}, B_{t}^{x}=\xi_{r}(x)$, 则 $B_{t}^{x}$ 是 Brown 运动 (定理 13(i)). 基于此结论, 一方面, 对给定 $x$, 当 $t \rightarrow \infty$ 时, 由重对数律, $B_{t}^{x}$ 的阶为 $\sqrt{t \log \log t}$, 于是,

$$
\mathbb{P}\left(\lim _{r \rightarrow 0} \frac{\xi_{r}(x)}{\log \frac{1}{r}}=0\right)=1, \quad \forall x .
$$

另一方面, $\mathbb{P}\left(B_{t}^{x} \geqslant \alpha t\right)=\mathbb{P}\left(B_{t}^{x} / \sqrt{t} \geqslant \alpha \sqrt{t}\right)$ 的阶为 $\mathrm{e}^{-\frac{1}{2} \alpha^{2} t}$. 因此, 当我们将 $D$ 划分为 $r^{-2}$ 个边长为 $r$ 的小方块时, 平均有 $O\left(r^{-2} \times \mathrm{e}^{-\frac{1}{2} \alpha^{2} t}\right)=O\left(r^{-\left(2-\frac{1}{2} \alpha^{2}\right)}\right)$ 个小方块满足: 中心 $x$ 的 $\xi_{r}(x) \geqslant \alpha \log \frac{1}{r}$. 从而, 中心点满足 $\xi_{r}(x) \approx \alpha \log \frac{1}{r}$ 的小方块平均有 $O\left(\left(\frac{1}{r}\right)^{2-\frac{1}{2} \alpha^{2}}-\left(\frac{1}{r}\right)^{2-\frac{1}{2}(\alpha+\varepsilon)^{2}}\right)=O\left(\left(\frac{1}{r}\right)^{2-\frac{1}{2} \alpha^{2}}\right)$ 个. 粗略地讲, 这意味着由 $\xi_{r}(x) \approx \alpha \log \frac{1}{r}$ 的 $x$ 组成的集合的维数为 $2-\frac{1}{2} \alpha^{2}$. 这即是定理 13(ii) 所刻画的现象.

定义 12 若 $\lim _{r \rightarrow 0} \xi_{r}(x) /(-\log r)=\alpha$, 则称 $x$ 为 $\xi$ 的一个 $\alpha$ - 厚点 (thick point). 将全体 $\alpha$ - 厚 点组成的集合记为 $T_{\alpha, \xi}$.

定理 13 (Brown 运动和厚点, 参见文献 [5, 定理 1.21] 和 [20, 定理 1.2]) 假设 $B\left(x, r_{0}\right) \subset D$, 令 $r=\mathrm{e}^{-t}, t_{0}=-\log r_{0}$, 则下面两条成立:

(i) $B_{t}^{x}:=\xi_{r}(x)\left(\forall t \geqslant t_{0}\right)$ 是 Brown 运动, 其中时空初值为 $\left(t_{0}, B_{t_{0}}^{x}\right)$.

(ii) 对任意 $0 \leqslant \alpha \leqslant 2, T_{\alpha, \xi}$ 的 Hausdorff 维数几乎必然为 $2-\alpha^{2} / 2$. 对任意 $\alpha>2, T_{\alpha, \xi}$ 几乎必然 为空集.

注 14 文献 [20] 考虑的是 $\eta(=\xi / \sqrt{2 \pi})$. 令 $\beta=\alpha^{2} / 2$. 该文献中 $\beta$ - 厚点指满足 $\eta_{r}(x) /(-\log r)$ $\approx \sqrt{\beta / \pi}$ 的点 (即定理 13 中的 $\alpha$ - 厚点), 其维数为 $2-\beta$ (即 $2-\alpha^{2} / 2$ ).

最后, 我们介绍二维 Gauss 自由场的共形不变性, 读者可参见文献 [5, 定理 1.19].

定理 15 (共形不变性) 假设 $\tilde{D}$ 也是 $\mathbb{R}^{2}$ 中的区域, $\varphi: D \rightarrow \tilde{D}$ 是共形映射, 那么, $\tilde{\xi}:=\xi \circ \varphi^{-1}$ 定义了 $\tilde{D}$ 上的一个随机分布, 它就是 $\tilde{D}$ 上的 Gauss 自由场.

共形不变性成立的原因是, 从 Gauss 自由场的随机函数意义看, 二维 Brown 运动的 Green 函数 具有共形不变性, 即 $G_{D}(x, y)=G_{\tilde{D}}(\varphi(x), \varphi(y))$; 从 Gauss 自由场的随机分布意义看, 如果 $\varphi$ 是共形 映射, 那么 $\int_{\tilde{D}} \nabla f(y) \cdot \nabla g(y) d y=\int_{D} \nabla(f \circ \varphi)(x) \cdot \nabla(g \circ \varphi)(x) d x$. 需强调的是, 当空间维数 $d \neq 2$ 时, 右 
边的积分中会出现 $\varphi$ 对应的 Jacobi 行列式的 $d-2$ 次方, 因此, 共形不变性是二维 Gauss 自由场特有 的性质.

\subsection{Liouville 量子引力场}

Liouville 量子引力场 (Liouville quantum gravity) 是物理学家 Polyakov 在 20 世纪 80 年代为研 究弦理论提出的模型, 其数学表达为一个二维随机曲面, 张量形如 $\mathrm{e}^{\gamma \xi(x)} d x$, 其中 $\gamma(>0)$ 是温度的倒 数, $\xi$ 是二维 Gauss 自由场. 如上节所述, $\xi$ 可视为一个零均值的 Gauss 场 $\{\xi(x), x \in D\}$, 其协方差函 数 $G(x, y)$ 由 (3.1) 给出. 虽然它的样本不是函数 (因此张量 $\mathrm{e}^{\gamma \xi(x)} d x$ 没有严格的数学定义), 但是在下 文中我们仍然经常将 $\xi$ 视为随机函数, 并做一些形式推导, 它们的严格证明请见相关文献. 目前, 人们 有几个切入点研究该随机曲面的几何结构, 它们是曲面体积 (即 Liouville 测度)、曲面上的 Brown 运 动 (即 Liouville Brown 运动) 和曲面距离. 我们将重点介绍研究得相当完善的 Liouville 测度和比较完 善的 Liouville Brown 运动. 对于研究刚起步的曲面距离, 我们不做介绍, 有兴趣的读者可以参见 Miller 和 Sheffield ${ }^{[21]}$ 的一系列新成果.

(1) Liouville 测度. 将协方差函数 $G(\cdot, \cdot)$ 满足 (3.2) 的零均值 Gauss 场称为对数相关的 Gauss 场, 其中 $C(\cdot, \cdot)$ 是有界连续函数. 对这样的 Gauss 场, 可以构造一个随机测度,

$$
\mu_{\gamma, \xi}(d x):=\exp \left(\gamma \xi(x)-\frac{1}{2} \gamma^{2} \mathbb{E} \xi(x)^{2}\right) d x
$$

它的期望是 Lebesgue 测度. 特别地, 当 $\xi$ 是 Gauss 自由场时, $\mu_{\gamma, \xi}$ 称为 Liouville 测度.

关于 $\mu_{\gamma, \xi}$ 的定义, 最早的研究可追溯到文献 [22], 方法是通过 $\xi$ 的光滑截断 (cut-off) 来逼近. 具 体地, 将 $G(\cdot, \cdot)$ 分解成协方差函数 $G_{n}(x, y)$ 之和, 即 $G(x, y)=\sum_{n} G_{n}(x, y)$. 取相互独立的零均值光滑 Gauss 场 $\zeta_{n}$ (光滑指 Gauss 场为随机函数), 以 $G_{n}(x, y)$ 为协方差函数, 然后证明 $\xi_{n}=\sum_{k=1}^{n} \zeta_{k}$ 对应 的 $\mu_{\gamma, \xi_{n}}$ 在 Radon 测度空间中几乎必然弱收敛, 其极限称为 $G(\cdot, \cdot)$ 对应的 Gauss 乘法噪声 (Gaussian multiplicative chaos). Gauss 乘法噪声在流体力学和经济学等领域有广泛的应用. $\mu_{\gamma, \xi_{n}}$ 收敛的本质原 因是, 对于任意 $A \subset D, \mu_{\gamma, \xi_{n}}(A)$ 是鞅 (因为 $\left\{\zeta_{k}\right\}$ 相互独立), 且当 $\gamma<2$ 时有 $\frac{4}{\gamma^{2}}-\varepsilon$ 阶矩 (见下面的 定理 18). 特别地, 当 $\gamma^{2}<2$ 时, 不难看出其二阶矩有限:

$$
\begin{aligned}
\mathbb{E} \mu_{\gamma, \xi_{n}}(A)^{2} & =\iint_{x, y \in A} \mathbb{E} \mathrm{e}^{\gamma\left(\xi_{n}(x)+\xi_{n}(y)\right)-\frac{1}{2} \gamma^{2}\left(\mathbb{E} \xi_{n}(x)^{2}+\mathbb{E} \xi_{n}(y)^{2}\right)} d x d y=\iint_{x, y \in A} \mathbb{E} \mathrm{e}^{\gamma^{2} \mathbb{E} \xi_{n}(x) \xi_{n}(y)} d x d y \\
& \leqslant \iint_{x, y \in A} \mathrm{e}^{\gamma^{2}(-\log \|x-y\|+C(x, y))} d x d y \leqslant C_{\gamma} \iint_{x, y \in A} \frac{1}{\|x-y\| \|^{2}} d x d y,
\end{aligned}
$$

其中 $C_{\gamma}$ 是常数.

另一种现在常用的构造是, 利用 Gauss 场的平均值进行逼近. 如前, 将 $\xi$ 在 $\partial B(x, \varepsilon)$ 上的平均值 记为 $\xi_{\varepsilon}(x)$, 并称之为 $\xi$ 的光滑化. 事实上, $\xi_{\varepsilon}$ 还可以取 $\xi$ 的其他光滑化. 可以证明, 当 $\varepsilon \rightarrow 0$ 时, $\mu_{\gamma, \xi_{\varepsilon}}$ 收玫. 需要指出的是, 上述极限一致, 且它不依赖于 $\xi_{\varepsilon}$ 和 $\xi_{n}$ 的选取. 该极限就是我们所寻的 $\mu_{\gamma, \xi}$.

定理 16 (Gauss 乘法噪声, 参见文献 [23, 定理 2.5]、 [24, 定理 2.1] 和 [25, 命题 1.1 和 1.2]) 假 设 $\xi$ 是 Gauss 自由场 (或更一般地, 对数相关的 Gauss 场), 则下面的极限相同, 且当 $\gamma<2$ 时该极限 不是零测度.

(i) 假设 $\left\{\xi_{n}\right\}$ 是 $\xi$ 的截断逼近, 那么, 当 $n \rightarrow \infty$ 时, 随机测度 $\mu_{\gamma, \xi_{n}}$ 几乎必然弱收玫;

(ii) 假设 $\left\{\xi_{\varepsilon}\right\}$ 是 $\xi$ 的光滑化逼近, 那么, 当 $\varepsilon \rightarrow 0$ 时, 随机测度 $\mu_{\gamma, \xi_{\varepsilon}}$ 依分布收玫. 
下面假设 $\gamma<2, \xi$ 特指 Gauss 自由场. 我们将介绍一系列结论, 它们从不同的角度体现 Liouville 量子引力场的分形结构. 首先需要指出的是, Liouville 测度的支撑是 $\gamma$ - 厚点集 $T_{\gamma, \xi}$. (形式运算的) 理由 如下: 考虑测度 $d \mu_{\gamma, h}(x) \mathbb{P}(d h)$, 其中 $\mathbb{P}$ 是 $\xi$ 的分布, $h$ 是 $\xi$ 的一个样本. 它可以写为 $C_{x} Z_{x} \tilde{\mathbb{P}}_{x}(d h) d x$, 其 中 $C_{x}=\mathrm{e}^{-\frac{1}{2} \gamma^{2} \mathbb{E} \xi(x)^{2}}, \tilde{\mathbb{P}}_{x}(d h)=\frac{1}{Z_{x}} \mathrm{e}^{\gamma h(x)} \mathbb{P}(d h), Z_{x}$ 为使得 $\tilde{\mathbb{P}}_{x}$ 为概率测度的归一化常数. 根据 Girsanov 变换, $\tilde{\mathbb{P}}$ 就是 $\{\tilde{\xi}(y)+\gamma G(x, y), y \in D\}$ 的分布, 其中 $\tilde{\xi}$ 也是 Gauss 自由场. 于是,

$$
\xi_{r}(x)=\tilde{\xi}_{r}(x)+\int_{\|y-x\|=r} \gamma G(x, y) d y=\tilde{\xi}_{r}(x)+\gamma \log \frac{1}{r}+O(1) .
$$

对 $\tilde{\xi}$ 应用 (3.5) 便知 $x$ 是 $\xi$ 的 $\gamma$ - 厚点. 这表明 $\left\{(x, h): x \in T_{\gamma, h}\right\}$ 在 $\mu_{\gamma, h}(d x) \mathbb{P}(d h)$ 下是满测度集 (即 其补集为零测集). 关于支撑集, 严格的推导可参见文献 [5, 定理 2.4].

定理 $\mathbf{1 7}$ (支撑集) $\mu_{\gamma, \xi}\left(T_{\gamma, \xi}^{c}\right)=0$.

根据 (3.2) 知, $G(r x, r y)=\log \frac{1}{r}+G(x, y)+O(1), \forall r>0$. 这表明在 $B(z, 1)$ 上, $\{\xi(z+r y)\}_{y \in B(0,1)}$ $\stackrel{d}{\approx}\{\xi(z+y)+Z\}_{y \in B(0,1)}$, 其中 $Z \sim N\left(0, \log \frac{1}{r}\right)$ 且 $Z$ 与 $\xi$ 相互独立. 于是, 若 $q$ 阶矩存在, 则

$$
\begin{aligned}
\mathbb{E} \mu_{\gamma, \xi}(B(z, r))^{q} & =\mathbb{E}\left(\int_{B(0, r)} \mathrm{e}^{\gamma \xi(z+y)-\frac{1}{2} \gamma^{2} \mathbb{E} \xi(z+y)^{2}} d y\right)^{q}=\mathbb{E}\left(\int_{B(0,1)} \mathrm{e}^{\gamma \xi(z+r y)-\frac{1}{2} \gamma^{2} \mathbb{E} \xi(z+r y)^{2}} r^{2} d y\right)^{q} \\
& \approx r^{2 q} \mathbb{E}\left(\left(\mathrm{e}^{\gamma Z-\frac{1}{2} \gamma^{2} \mathbb{E} Z^{2}}\right)^{q}\left(\int_{B(0,1)} \mathrm{e}^{\gamma \xi(z+y)-\frac{1}{2} \gamma^{2} \mathbb{E} \xi(z+y)^{2}} d y\right)^{q}\right) \\
& =r^{2 q} \mathbb{E} \mathrm{e}^{q \gamma Z-\frac{1}{2} q \gamma^{2} \mathbb{E} Z^{2}} \mathbb{E} \mu_{\gamma, \xi}(B(0,1))^{q}=C_{q} r^{\alpha_{\gamma}(q)},
\end{aligned}
$$

其中 $C_{q}=\mathbb{E} \mu_{\gamma, \xi}(B(0,1))^{q}$, 我们用到 $Z$ 与 $\xi$ 相互独立, $Z \sim N\left(0, \log \frac{1}{r}\right)$, 从而,

$$
\mathbb{E} \mathrm{e}^{q \gamma Z-\frac{1}{2} q \gamma^{2} \mathbb{E} Z^{2}}=r^{-\frac{1}{2} q^{2} \gamma^{2}+\frac{1}{2} q \gamma^{2}}, \quad \alpha_{\gamma}(q)=\left(2+\frac{\gamma^{2}}{2}\right) q-\frac{\gamma^{2}}{2} q^{2} .
$$

当 $\gamma \neq 0$ 时, $\alpha_{\gamma}(q)$ 不是 $q$ 的线性函数, 这说明 Liouville 测度有重分形结构, $\alpha_{\gamma}(q)$ 称为重分形谱 (multifractal spectrum) 或结构函数 (structure function).

定理 18 (矩, 参见文献 [24, 命题 3.5-3.7]) 记 $q_{*}=\frac{4}{\gamma^{2}}$, 那么下面两条成立:

(i) 若 $q<q_{*}$, 则当 $r \rightarrow 0$ 时, $\mathbb{E} \mu_{\gamma, \xi}(B(z, r))^{q} \approx C_{q} r^{\alpha_{\gamma}(q)}$.

(ii) 当 $q \in\left(0, q_{*}\right)$ 时, 对任意 $A \subset D$, 有 $\mathbb{E} \mu_{\gamma, \xi}(A)^{q}<\infty$, 且 $\lim _{\varepsilon \rightarrow 0} \mathbb{E} \mu_{\gamma, \xi_{\varepsilon}}(A)^{q}=\mathbb{E} \mu_{\gamma, \xi}(A)^{q}$, 其中 $\xi_{\varepsilon}$ 同定理 16. 当 $q \leqslant 0$ 时, 上述结论对 $A=B(z, r)$ 成立.

注 19 一般地, 对于 $d$ 维 Gauss 自由场, 当 $\lambda^{2}<2 d$ 时, 记 $q_{*}$ 为 $\left(d+\frac{\gamma^{2}}{2}\right) q-\frac{\gamma^{2}}{2} q^{2}=d$ 在 $q \in(1, \infty)$ 上唯一的根, 则上述结论仍然成立 (参见文献 [24]).

下面介绍著名的 KPZ 关系式 (根据三位物理学家 Knizhnik、Polyakov 和 Zamolodchikov 命名, 参 见文献 [26]), 它刻画了一个集合在欧氏空间的维数与在量子空间的维数之间的关系, 其中, 在欧氏空 间中使用 Lebesgue 测度 $\mu_{0, \xi}$, 在量子空间中使用 Liouville 测度 $\mu_{\gamma, \xi}$. 假设 $B_{\gamma}(x, \varepsilon)$ 是以 $x$ 为中心的 圆盘, 其 $\mu_{\gamma, \xi}$ 测度为 $\varepsilon$. 当 $\gamma=0$ 时, $B_{0}(x, \varepsilon)$ 的半径是 $\sqrt{\varepsilon / \pi}$. 对集合 $A \subset D$, 记

$$
B_{\gamma}(A, \varepsilon):=\left\{x: B_{\gamma}(x, \varepsilon) \cap A \neq \emptyset\right\}, \quad \Delta_{\gamma}=\lim _{\varepsilon \rightarrow 0} \frac{1}{\log \varepsilon} \log \mathbb{E} \mu_{\gamma, \xi}\left(B_{\gamma}(A, \varepsilon)\right) .
$$

$\Delta_{\gamma}$ 称为标度指数 (scaling exponent). 在 $\gamma=0$ 和 $\gamma>0$ 的情形, $\Delta_{\gamma}$ 分别称为欧氏标度指数和量子标 度指数. 下面的 $\mathrm{KPZ}$ 关系式描述了集合的欧氏标度指数与量子标度指数之间的关系.

定理 $20\left(\mathrm{KPZ}\right.$ 关系式, 参见文献 [25, 定理 1.4]) $\quad \Delta_{0}=\frac{\gamma^{2}}{4} \Delta_{\gamma}^{2}+\left(1-\frac{\gamma^{2}}{4}\right) \Delta_{\gamma}$. 
我们从 (Minkowski) 维数的角度简要指出支持 $\mathrm{KPZ}$ 关系式成立的一个理由. 为方便起见, 我们用 边长为 $r$ 的小方块代替圆盘. 把空间划分为边长为 $r=2^{-n}$ 的小方块, 把这些小方块记为 $\mathcal{B}_{n}$. 将集合 $A$ 的 (在参数为 $\gamma$ 时的) 维数定义为

$$
d_{\gamma}=\inf \left\{d: \limsup _{n \rightarrow \infty} \sum_{B \in \mathcal{B}_{n}, B \cap A \neq \emptyset} \mu_{\gamma, \xi}\left(B_{i}\right)^{d}<\infty\right\} .
$$

这表明, 当我们用 $\mu_{\gamma, \xi}$ 体积约为 $\varepsilon$ 的小方块覆盖 $A$ 时, 需要的小方块数约为 $\left(\frac{1}{\varepsilon}\right)^{d_{\gamma}}$, 于是,

$$
\Delta_{\gamma} \approx \frac{1}{\log \varepsilon} \log \left(\left(\frac{1}{\varepsilon}\right)^{d_{\gamma}} \times \varepsilon\right)=1-d_{\gamma} .
$$

因此, 我们只需要找出 $d_{0}$ 与 $d_{\gamma}$ 的关系即可, 这一点可由定理 18 得到,

$$
\mathbb{E} \sum_{B \in \mathcal{B}_{n}, B \cap A \neq \emptyset} \mu_{\gamma, \xi}\left(B_{i}\right)^{d}=O\left(\sum_{B \in \mathcal{B}_{n}, B \cap A \neq \emptyset} \mu_{0, \xi}\left(B_{i}\right)^{\frac{1}{2} \alpha_{\gamma}(d)}\right),
$$

其中我们用到 $\mu_{0, \xi}$ 是 Lebesgue 测度, 边长为 $r$ 的小方块的 Lebesgue 测度的阶为 $r^{2}$. 这表明 $\frac{1}{2} \alpha_{\gamma}\left(d_{\gamma}\right)$ $=d_{0}$, 这等价于 $\mathrm{KPZ}$ 关系式.

(2) Liouville Brown 运动及其热核. 仿照流形上的 Brown 运动的定义, Liouville Brown 运动可以 通过对坐标空间 (欧氏空间) 中的 Brown 运动做时间变换得到. 时间变换的函数为

$$
F_{\gamma, \xi}(t)=\int_{0}^{t} \exp \left(\gamma \xi\left(B_{s}\right)-\frac{1}{2} \gamma^{2} \mathbb{E} \xi\left(B_{s}\right)^{2}\right) d s,
$$

其中 $\left\{B_{s}\right\}$ 是 $\mathbb{R}^{2}$ 上的标准 Brown 运动.

它也是通过截断构造的. 为给出其严格定义, 我们需要取定理 16 中的 $\xi_{n}$ 作为截断.

定理 21 (Liouville Brown 运动和热核, 参见文献 [27, 定理 2.7 和 2.18]、[28, 定理 0.4] 和 [29, 定 理 1.2 和 1.3]) 给定 $\gamma \in(0,2)$. 对几乎所有的 Gauss 场样本, 下面三条成立:

(i) $F_{\gamma, \xi_{n}}(\cdot)$ 依 $\left(\left\{B_{s}\right\}\right.$ 的) 概率收玫, 记其极限为 $F_{\gamma, \xi}(\cdot)$. 记 $\mathcal{B}_{t}=B_{F^{-1}(t)}$, 则 $\left\{\mathcal{B}_{t}\right\}_{t \geqslant 0}$ 是轨道连续 的强马氏过程, 其转移半群记为 $P_{t}^{\gamma}$.

(ii) 存在对称的非负密度函数 $p_{t}^{\gamma}(x, y)$, 使得 $P_{t}^{\gamma} f(x)=\int_{D} f(y) p_{t}^{\gamma}(x, y) \mu_{\gamma}(d y)$, 其中 $\mu_{\gamma}$ 是 Liouville 测度.

(iii) 对任意 $\beta>(\gamma+2)^{2} / 2$, 存在常数 $C_{1}$ 和 $C_{2}$ 使得 $p_{t}^{\gamma}(x, y) \leqslant C_{1} \exp \left(-C_{2} t^{-\frac{1}{\beta-1}}\right), \forall t \in(0,1 / 2]$; 存在常数 $C_{3}>0$ 使得 $p_{t}(x, x) \geqslant C_{3} \frac{1}{t}\left(\log \frac{1}{t}\right)^{-34}(\forall t)$ 充分小.

利用 Brown 运动的尺度变换, 可以仿照定理 18(i), 证明

$$
\mathbb{E}(F(t+s)-F(t))^{q} \leqslant C_{q} s^{\alpha_{\gamma}(q)} .
$$

上述定理中的 $\left\{\mathcal{B}_{t}\right\}$ 称为 Liouville Brown 运动, $p_{t}^{\gamma}(x, y)$ 称为 Liouville 热核. 热核是研究曲面的几何结 构的重要参量, 也是研究 KPZ 关系式的一个工具 (参见文献 [30]).

\section{3 一维和高维 Gauss 自由场}

当 $d=1$ 时, 区间上的 Gauss 自由场就是 Brown 桥 (Dirichlet 边界条件) 或 Brown 运动 (其他边 界条件). 此时, Brown 桥的 Fourier 级数就是 $\eta$ 在 $H_{0}^{1}(D)$ 中的分解, Gauss 乘法噪声可应用于刻画物 理中的湍流 ${ }^{[22]}$ 和经济学中的资产价格波动 ${ }^{[24]}$. 
下面假设 $d \geqslant 3$. 由于 Brown 运动是非常返的, 在定义 Gauss 自由场时可以不要求 $D$ 是有界区域, 也可以不进行边界截杀. 对于离散型 Gauss 自由场 $\xi$, 其协方差函数为 $G(u, v)=\mathbb{E}_{u} \sum_{n=0}^{\infty} \mathbb{P}\left(S_{n}=v\right)$, $\forall u, v \in \mathbb{Z}^{d}$. 给定高度 $h$, 点集 $E_{h}:=\left\{u \in \mathbb{Z}^{d}: \xi_{u} \geqslant h\right\}$ 称为游式集 (excursion set). 若能在 $E_{h}$ 中找到 路径连接 $u$ 和 $v$ (或集合 $A$ 到 $B$ ), 则记为 $u \stackrel{\geqslant}{\longleftrightarrow} v$ (或 $A \stackrel{\gtrless}{\longleftrightarrow} B$ ). 借助渗流的语言, 若 $\xi_{u} \geqslant h$, 则称 $u$ 为 “开”, 否则称 $u$ 为 “闭”. 那么, $0 \stackrel{\geqslant}{\longleftrightarrow} \infty$ 意味着包含顶点 0 的开簇是一个无穷开簇. 根据 $E_{h}$ 关于 $h$ 的单调性, 令

$$
\begin{aligned}
& h_{*}(d):=\inf \{h \in \mathbb{R}: \mathbb{P}(0 \stackrel{\geqslant h}{\longleftrightarrow} \infty)=0\}, \\
& h_{* *}(d):=\inf \left\{h \in \mathbb{R}: \text { 存在 } \alpha>0 \text { 使得 } \lim _{L \rightarrow \infty} L^{\alpha} \mathbb{P}(B(0, L) \stackrel{\gtrless h}{\longleftrightarrow} S(0,2 L))=0\right\},
\end{aligned}
$$

其中 $S(0,2 L)$ 是以原点为中心、 $2 L$ 为边长的方盒. 显然, $h_{*}(d) \leqslant h_{* *}(d)$. 下面的结论给出 $\mathbb{Z}^{d}$ 上 Gauss 自由场的临界值的估计, 对于齐次树上的 Gauss 自由场, 相应的估计参见文献 [31].

定理 22 (参见文献 [32, 定理 2.6 和 3.3] 和 [33, 定理 0.1-0.3]) 下面两条成立:

(i) 对任意 $d \geqslant 3, h_{* *}(d)<\infty$ (从而 $h_{*}(d)<\infty$ ), 且当 $h>h_{* *}(d)$ 时, 存在 $\alpha_{h}, \beta_{h}>0,0<\rho<1$ 使得 $\mathbb{P}(B(0, L) \stackrel{\geqslant h}{\longleftrightarrow} S(0,2 L)) \leqslant \alpha_{h} \exp \left(-\beta_{h} L^{\rho}\right)$. 存在 $d_{0}$ 使得当 $d \geqslant d_{0}$ 时, $h_{*}(d)>0$.

(ii) 当 $d \rightarrow \infty$ 时, $\mathbb{P}\left(\xi_{0} \geqslant h_{*}(d)\right)=\frac{1}{d^{1+o(1)}}$. 令 $h(d)=\sqrt{2 G(0,0) \log d}$, 则 $h_{*}(d) \sim h_{* *}(d) \sim h(d)$, 其 中 $\alpha_{d} \sim \beta_{d}$ 指 $\lim _{d \rightarrow \infty} \alpha_{d} / \beta_{d}=1$.

此外, 对于 $d=4$, 文献 [34] 研究了厚点的维数; 文献 [35] 研究了 Gauss 自由场的 KPZ 关系式.

\section{参考文献}

1 Kenyon R. Dominos and the Gaussian free field. Ann Probab, 2001, 29: 1128-1137

2 Rider B, Virag B. The noise in the circular law and the Gaussian free field. Int Math Res Not IMRN, 2007, 2007: rnm006

3 Zeitouni O. Gaussian fields. Http://www.wisdom.weizmann.ac.il/ zeitouni/notesGauss.pdf, 2016

4 Lyons R, Peres Y. Probability on Trees and Networks. Cambridge: Cambridge University Press, 2017

5 Berestycki N. Introduction to the Gaussian free field and Liouville quantum gravity. Http://www.statslab.cam.ac. uk/ beresty/Articles/oxford4.pdf, 2016

6 Bolthausen E, Deuschel J D, Giacomin G. Entropic repulsion and the maximum of the two-dimensional harmonic crystal. Ann Probab, 2001, 29: 1670-1692

7 Bramson M, Zeitouni O. Tightness of the recentered maximum of the two-dimensional discrete Gaussian free field. Comm Pure Appl Math, 2012, 65: 1-20

8 Bramson M, Ding J, Zeitouni O. Convergence in law of the maximum of the two-dimensional discrete Gaussian free field. Comm Pure Appl Math, 2015, 69: 62-123

9 Aïdékon E. Convergence in law of the minimum of a branching random walk. Ann Probab, 2013, 41: 1362-1426

10 Chatterjee S, Dembo A, Ding J. On level sets of Gaussian fields. ArXiv:1310.5175, 2013

11 Daviaud O. Extremes of the discrete two-dimensional Gaussian free field. Ann Probab, 2006, 34: 962-986

12 Biskup M, Louidor O. Extreme local extrema of two-dimensional discrete Gaussian free field. Comm Math Phys, 2016, 345: 271-304

13 Ding J, Goswami S. Upper bounds on Liouville first passage percolation and Watabiki's prediction. ArXiv:1610.09998, 2016

14 Ding J, Zhang F. Liouville first passage percolation: Geodesic dimension is strictly larger than 1 at high temperatures. ArXiv:1610.02766, 2016

15 Eisenbaum N, Kaspi H, Marcus M B, et al. A Ray-Knight theorem for symmetric Markov process. Ann Probab, 2000, 28: $1781-1796$

16 Lupu T. From loop clusters and random interlacements to the free field. Ann Probab, 2016, 44: 2117-2146

17 Zhai A. Exponential concentration of cover times. ArXiv:1407.7617, 2014 
18 Schramm O, Sheffield S. Contour lines of the two-dimensional discrete Gaussian free field. Acta Math, 2009, 202: $21-137$

19 Sheffield S. Gaussian free fields for mathematicians. Probab Theory Related Fields, 2007, 139: 521-541

$20 \mathrm{Hu}$ X, Miller J, Peres Y. Thick points of the Gaussian free field. Ann Probab, 2010, 38: 896-926

21 Miller J, Sheffield S. Liouville quantum gravity and the Brownian map I, II and III. ArXiv:1607.00719, 2015; arXiv:1605.03563, 2016; arXiv:1608.05391, 2016

22 Kahane J P. Sur le chaos multiplicatif. Ann Sci Math Québec, 1985, 9: 105-150

23 Robert R, Vargas V. Gaussian multiplicative chaos and applications: A review. Probab Surv, 2014, 11: 315-392

24 Robert R, Vargas V. Gaussian multiplicative chaos revisited. Ann Probab, 2010, 38: 605-631

25 Duplantier B, Sheffield S. Liouville quantum gravity and KPZ. Invent Math, 2011, 185: 333-393

26 Knizhnik V G, Polyakov A M, Zamolodchikov A B. Fractal structure of 2D-quantum gravity. Modern Phys Lett A, 1988, 3: 819-826

27 Garban C, Rhodes R, Vargas V. Liouville Brownian motion. Ann Probab, 2016, 44: 3076-3110

28 Garban C, Rhodes R, Vargas V. On the heat kernel and the Dirichlet form of Liouville Brownian motion. Electron J Probab, 2014, 19: 1-25

29 Andres S, Kajino N. Continuity and estimates of the Liouville heat kernel with applications to spectral dimensions. Probab Theory Related Fields, 2016, 166: 713-752

30 Berestycki N, Garban C, Rhodes R, et al. KPZ formula derived from Liouville heat kernel. J Lond Math Soc (2), 2016, 94: 186-208

31 Sznitman A S. Coupling and an application to level-set percolation of the Gaussian free field. Electron J Probab, 2016, 21: $1-26$

32 Rodriguez P F, Sznitman A S. Phase transition and level-set percolation for the Gaussian free field. Comm Math Phys, 2013, 320: 571-601

33 Drewitz A, Rodriguez P F. High-dimensional asymptotics for percolation of Gaussian free field level sets. Electron J Probab, 2015, 20: 1-39

34 Cipriani A, Hazra R S. Thick points for a Gaussian free field in 4 dimensions. Stochastic Process Appl, 2015, 125: 2383-2404

35 Chen L, Jakobson D. Gaussian free fields and KPZ relation in $\mathbb{R}^{4}$. Ann Inst H Poincaré, 2014, 15: 1245-1283

\section{Gaussian free field and related fields}

\section{CHEN DaYue \& ZHANG FuXi}

Abstract In this paper, we review the development of Gaussian free fields in recent years, including equivalent definitions, elegent properties, as well as delicate structures. We also introduce the Liouville quantum gravity (random surface) based on Gaussian free fields, including Liouville measure and Liouville Brownian motion.

Keywords Gaussian free field, log-correlated Gaussian field, Liouville quantum gravity

$\operatorname{MSC}(2010) \quad 60 \mathrm{G} 60,60 \mathrm{~K} 35$

doi: 10.1360/N012017-00128 